\title{
The Range of European Nutritional Practice - Inspiring and on the Point
}

\section{The Czech Association of Dietitians \\ New Czech National Standards of Hospital Food and Nutrition Care}

After nearly three decades the Czech Ministry of Health issued new National Standards of Hospital Food and Nutrition Care. Notably, this is the first time that the National Standards contains chapters dedicated to nutrition care.

While nutrition care standards have been developed by many Czech hospitals, a nutrition care standard on a national level did not exist and the national standard of hos-

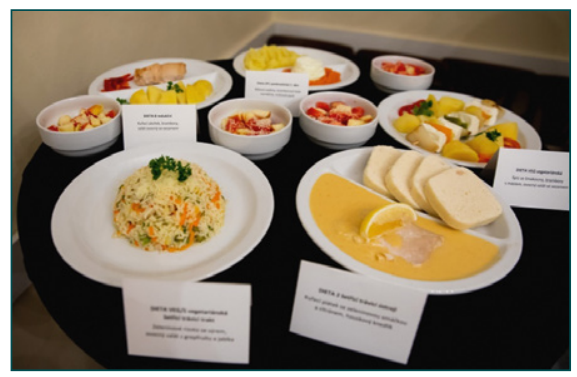

Samples of Czech traditional hospital meals. pital food was last revised in 1991. Therefore in 2019, the Minister of Health initiated work on a new national standard under the professional supervision of SKVIMP (CzechSPEN) and the (zech Association of Dietitians (CAD), and mediated the cooperation with over 20 hospitals.

The new standard is a principle-based document which aims to help hospitals organize nutrition care in line with the ESPEN guidelines and brings a simplified overview of hospital diets and their indications. Most importantly, it includes guidance on a basic diet which respects nutrition guidelines for a healthy population.

During the development phase it became clear that while new chapters on nutrition care are widely accepted, implementation of the new hospital food standard will be a challenge. A survey among hospitals found that $68 \%$ of the respondents still manage food service based on the system developed in 1955 which followed a Soviet pattern of numbered diets for groups of patients. The 1955 system was well supported by detailed publication containing diet principles, energy and nutrient intake recommendations along with hundreds of recipes. However, it is not based on current scientific evidence and promotes limiting nutrients for certain groups of patients, possibly resulting in malnutrition.

In order to promote acceptance of this new evidence-based document and enable its successful implementation, the Czech hospital food service needs a new vision for becoming a valued provider of patientcentred nutrition care.

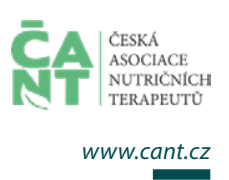

Hellenic Dietetic Association (HDA)

\section{Greece Is Officially the 19th Member of the Optimal Nutrition Care for All (ONCA) Campaign}

This European initiative led by the European Nutrition Health Alliance (ENHA) will facilitate processes regarding the screening of disease-related malnutrition risk and will promote the implementation of appropriate nutritional care protocols for patients at national level.

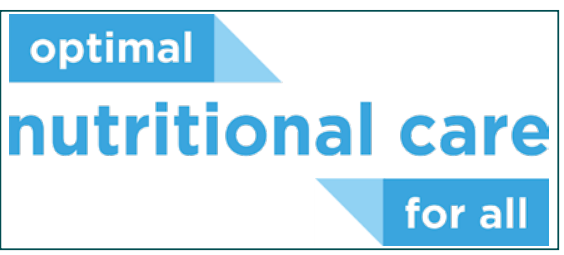

After coordinated efforts of the Hellenic Dietetic Association (HDA) and the Hellenic Society for Medical / Clinical Nutrition \& Metabolism (GrESPEN), Greece will actively participate in the design and implementation of the appropriate clinical practices for the evaluation and treatment of patients' nutritional status.

The main contributors to this effort were Dr. Christina Katsagoni, (President of the Clinical Nutrition Specialists group of HDA) and Dr. Dimitris Karagiannis (LLL program manager in Greece) assisted by
Dr. Theodora-Irini (Dorina) Sialvera (President of HDA) the Assist. Professor of Medical Nutrition Michael Chourdakis (President of GrESPEN) and the board members of the two scientific societies. 


\section{Improve the Future Through Food Sustainability}

Food has a considerable environmental impact, taking into account its production, transformation, transport and consumption practices, where it is possible to highlight the existing food waste in extremely high numbers, as it is known. In this way, the importance of reflecting on food sustainability is increasingly emphasized, not forgetting to join the environmental, social and economic axis, in order to contribute to the existence

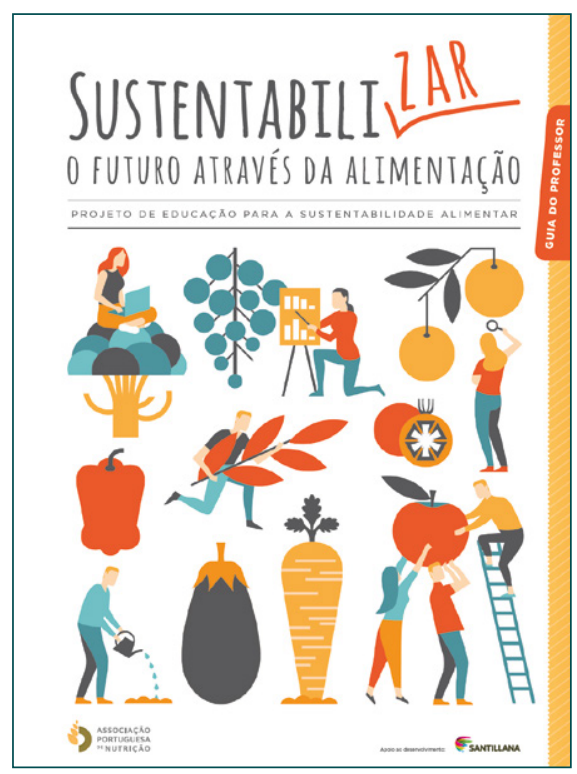

of eating habits with a lower environmental impact and that contribute to the food and nutritional security of the population, as well as for their state of health, both now and in the future. Our food must protect and respect biodiversity and the ecosystem, in addition to allowing the optimization of natural and human resources.

In this field, nutritionists can be professionals of excellence for the transmission of these messages, reinforcing and clarifying that a sustainable diet is healthy, but that a healthy diet may not be sustainable, making all the difference the choices we make in our daily lives (choose national, fresh, respect seasonality, minimize waste, recycle, etc.).

The Portuguese Association of Nutrition has been working on these themes in a food sustainability program since 2017, and is currently carrying out a project in secondary schools that aims to inform, raise awareness and put young people to reflect on their choices and whether they are sustainable. Providing young people, adults of tomorrow, with better knowledge, far from extremism and imbued with balance and consideration, will be one of the solutions to ensure that we meet present and future needs, without causing irreversible damage to the ecosystem and without compromising the future of the next generations. The project has been gaining good support from schools, with around 2500 students involved since the beginning of 2020. Improve the future through food sustainability is something that emerges and must be worked in a multidisciplinary way by the various agents in the food, health, education, environment, economy, among others. It is the order of the day and we should all feel involved, as only everyone's effort will make a difference.

Helena Real, Nutritionist

More information about the sustainability program of Portuguese Association of Nutrition at:

https://sustentabilidadealimentar.apn.org.pt/

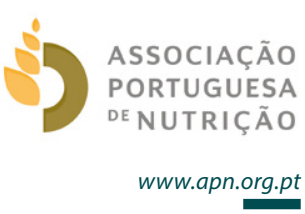

Ecotrophelia Trophy 2020

\section{The Future of Food Innovation}

OrangeBeeee it the big winner of of Ecotrophelia 2020. Two young women from the Unviversity of Aveiro, Portugal, have created a fermented preparation of aquafaba and yacon with a layer of orange peel jam, sprinkled with bee pollen. This product is a vegetarien alternative to joghurt. Further rewarded attendees were a team of University of the Aegean, Greece, with O-live, an ecological breadstick and two students of Iceland University. With Frosti they originate a lactose-free organic Icelandic dairy product and skyr flakes.

Established in France in 2000 and expanded to a European scale in 2008, ECOTROPHELIA Europe is organized by the European Technology Platform "Food for Life", ANIA (Na- tional Association of Food Industries) and CCI Vaucluse. On the principle of a food innovation "Champions League" each European country organizes its own national competition to select the most innovative food project that will then be presented at ECOTROPHELIA Europe. Each country selection is coordinated by its national food federation. The teams are composed of 2 to 10 students from either public or private higher education European establishments, scientific or commercial. The prize money this year was 9500 Euro.

Dominique Ladevèze, initiator of ECOTROPHELIA, highlights: "2020 is a complex year for everyone and our competition was no exception." Due to pandemic reasons, 13 teams presented their innovations in a video forum. "With a peak of more than 600 people connected", Ladevèze emphesizes. The jury was very much impressed by this hunger for innovation, which could have been slowed down by the pandemic. Well, not at all! "We are also noticing the inexorable rise of , healthy', , free', vegetarian and vegan trends, which lead us to lighter and healthier products - provided that taste and pleasure are met".

ECOTROPHELIA EUROPE

https://eu.ecotrophelia.org
32

Kompass Nutr Diet 2021;1:31-34 DOI: $10.1159 / 000513441$ 


\section{Hungarian Dietetic Association}

\section{Promoting the Dietetic Profession in Hungary}

The first Dietitian's Week in Hungary was held between 2nd and 6th of June 2020, with which the Hungarian Dietetic Association showed the diversity of their profession and emphasized, how they can help develop proper nutrition and diets for the treatment of certain diseases. The aim of their Facebook campaign was to make the general public aware that it is worth contacting dietitians with questions related to nutrition because they are experts in this field. In Hungary, college education dates back to forty-five years, and a dietitian degree can be obtained after four years of study. The degree entitles you to three areas: prevention, nutrition management, and clinical dietetics. The professional organization of dietitians, the Hungarian Dietetic Association (MDOSZ), has been representing and bringing together the profession for almost thirty years. According to a survey from 2018, the majority of the Hungarian adult population (61\%) know what dietitians do and would turn to them primarily about their nutrition-related questions. The vast majority (89\%) consider the opinions and advice of dietitians to be the most authentic and understandable. However, for the word nutrition advice, Google will return 653,000 hits in a matter of seconds. The mass of information is difficult to adjust to, so they often turn to those without a dietetic education for advice, which is often very harmful.

Meanwhile, it has also been shown that closer integration of nutrition counseling into primary care could have a number of economic and public health benefits. Proper nutrition, based on scientific evidence, reduces the risk and development of dietrelated diseases, including obesity, diabetes, and cardiovascular disease, and could therefore reduce health care costs. International research shows that every Euro spent on nutrition advice saves 14-63 Euro to the society, not to mention an individual's health, quality of life, and well-being.

The Dietitians' Week initiative would like to raise the profile of dietetic practice as a pub-

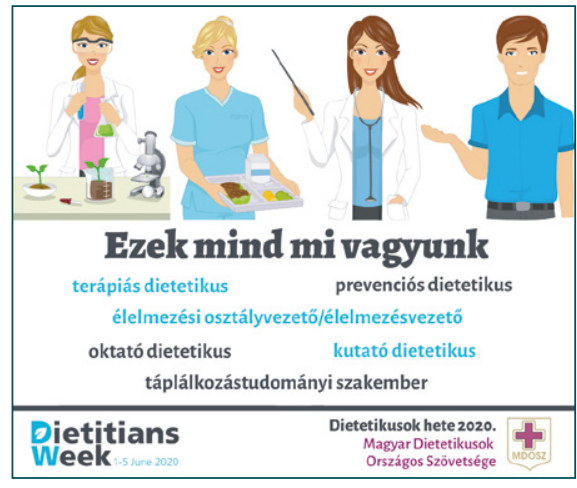

lic health importance. From the 2 nd of June 2020, for five days, the Hungarian Dietetic Association's Facebook page promoted the dietetic profession with informative posts and infographics.

The campaign (press release and SM activities) reached almost 1 million people and gained 27.000+ Euro earned media.

Zsuzsanna Zsüzs

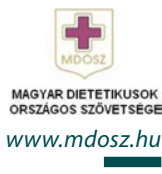

\section{Cochrane Review}

\section{Does a Tax on Fat in Food Help Against Overweight?}

A tax on high-fat foods could probably have a positive effect on people's diet. However there are still too few reliable studies. This is the conclusion of a study published in the Cochrane Database of Systematic Reviews by an international research team from the University of Bremen, the Danube University Krems, the AOK Baden-Württemberg and other international research institutions.

Denmark was the only country in the world to introduce a fat tax in the years 2011-2012. It was set at 16 Danish Kroner (a good two euros) per kilogram of saturated fatty acids

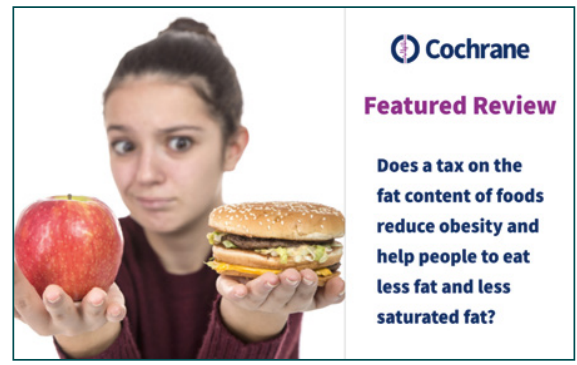

for all foods in which this type of fat accounted for more than $2.3 \%$ of the total weight. However, the first author of the current Cochrane Review, Dr. Stefan K. Lhachimi (University of Bremen) emphasizes that the shortlived Danish fat tax is a lost opportunity: "It is tragic that Denmark has failed to evaluate this legal measure in a meaningful way with regard to its health effects."

According to the two found studies the sales of cream decreased with the fat tax in Denmark by nearly $6 \%$ mincemeat had been in demand by $4 \%$ less. "A study reported a reduction in per capita fat consumption of 42 grams per week," said Dr. Stefan K. Lhachimi. However, this observation was based on purchases in supermarkets. "Unfortunately, we do not know what people actually ate: less, just as much or possibly even more fat, just from other sources."

A tax on saturated fatty acids like in Denmark could be a good approach to make so-called junk food more expensive and thus less attractive. "Everyone has a rough gut feeling about what junk food is," says Lhachimi, "but it is difficult to find a clear definition here under food law." A tax on unsaturated fatty acids would automatically affect many products from the junk food group. He critisizes the Danish fat tax as lost opportunity. As an active researcher it is sad to see that the accountable authorities did not implement a thorough prospective evaluation of this game-changing policy initiative.

\section{Literature}

Lhachimi SK, Pega F, Heise TL et al.: Taxation of the fat content of foods for reducing their consumption and preventing obesity or other adverse health outcomes. Cochrane Database of Systematic Reviews 2020, Issue 9. Art. No.: CD012415. DOI: 10.1002/14651858.CD012415.pub2.

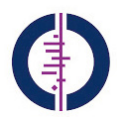

www.cochrane.org 


\section{Infection with SARS-CoV-2 via Pork Meat Unlikely According to Current State of Knowledge}

State media in China have claimed that a worker has become infected with the novel coronavirus (SARS-CoV-2) from a knuckle of pork imported from Germany. The infection is reported to have taken place in a cold store. Traces of SARS-CoV-2 were detected on packaging as well as on a door knob. According to the current state of knowledge, there are no cases that have shown evidence of humans being infected with the novel coronavirus via the consumption of contaminated food. Nor has any reliable evidence being presented to date concerning transmission of the virus via contact with contaminated objects or contaminated surfaces - such as packaging - which would have led to subsequent infections in humans.

According to the current state of knowledge, farm animals used for the production of meat cannot become infected with SARS-CoV-2 and are therefore unable to transmit the virus to humans via this pathway. Contamination of meat and meat products or its packaging with coronavirus could occur during the slaughtering, butchering, processing and packaging processes, however.
Generally, coronaviruses can potentially be transferred from an infected person to meat products if hygiene rules are not followed, - for example, by sneezing or coughing onto these products, or through contaminated hands. The same applies to surface contaminations (on packaging, for example). However, the hygiene rules and safety precautions that are commonly observed during the slaughtering, processing and packaging of meat minimise the risk of contamination with pathogens, which also applies for SARS-CoV-2.

Coronaviruses cannot multiply in or on food; they require a living animal or human host to do this. Transmission of the virus to another person via a contact infection appears possible only if this person touches a contaminated item of food or packaging and then transfers the virus to the mucous membranes of their nose or eyes with the hands. According to the current state of knowledge, the oral/alimentary route of transmission through the consumption of meat is not relevant for the current SARSCov-2 pandemic.

It is unclear whether the detected traces of virus are derived from an infectious virus or whether the virus had already been inactivated by storage or transportation. Nor do the reports state whether the traces of the virus were already present on the imported product or had been transferred to the packaging and door knob by the infected worker.

To protect yourself from viral infection, always observe the general rules for everyday hygiene: ensure that you wash your hands regularly and avoid touching your face with your hands - especially while preparing food. Furthermore, meat and poultry in general should be heated sufficiently and evenly before consumption, until the meat juice trickling out is clear and the meat is a whitish (poultry), greyish-pink (pork) or greyish-brown (beef) colour.

More information on hygiene when handling food can be found here:

https://www.bfr.bund.de/cm/364/protectionagainst-foodborne-infections.pdf

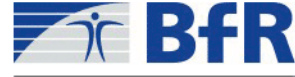
Bundesinstitut für Risikobewertung

www.bfr.bund.de/en

\section{News in}

\section{Kompass Nutrition \& Dietetics}

Dear Reader,

Please contribute news from national dietetic associations. For an easy handling please note:

\section{For Kaleidoscope we use press releases. Please submit \\ o an English text (not more than 2,000 characters) with a catchy headline \\ o full name of the institute/organization who spread the news, its logo and a web address}

Additionally, a photo, graphic or other eyecatcher could be included, as long as you hold the copyright and we can use the element for free.

\author{
For the Calendar of events \\ please submit \\ o name, \\ o date, \\ o location, \\ o website of the event
}

